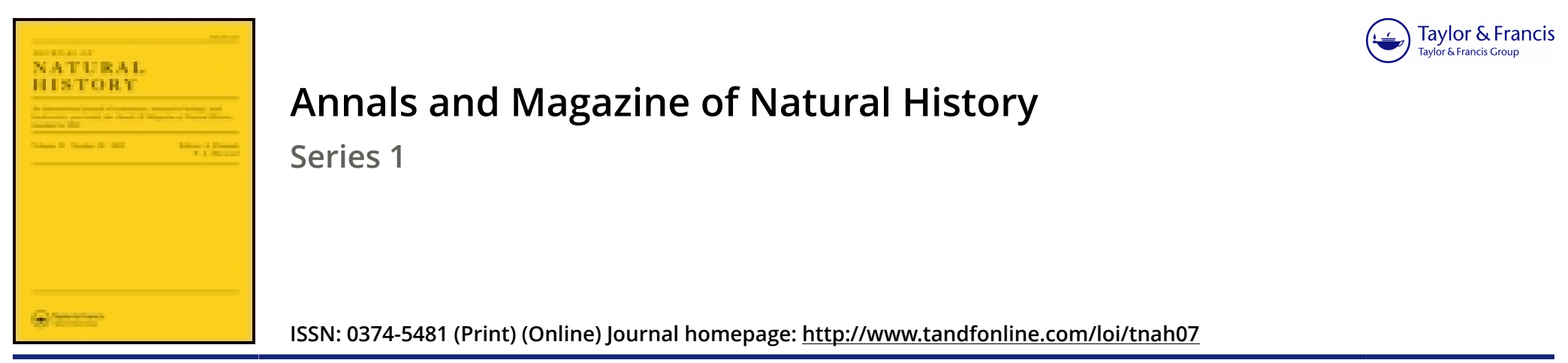

\title{
XLV.-An index to the British annelides
}

\section{George Johnston M.D. LL.D.}

To cite this article: George Johnston M.D. LL.D. (1846) XLV.-An index to the British annelides , Annals and Magazine of Natural History, 16:108, 433-462, DOI: 10.1080/037454809495980

To link to this article: http://dx.doi.org/10.1080/037454809495980

曲 Published online: 23 Dec 2009.

Submit your article to this journal 중

III Article views: 2

Q View related articles $₫$

Citing articles: 2 View citing articles 


\title{
THE ANNALS
}

AND

\section{MAGAZINE OF NATURAL HISTORY.}

\author{
SUPPLEMENT TO VOL. XVI. JANUARY 1846.
}

XLV.-An Index to the British Annelides. By George Johnston, M.D., LL.D., Fellow of the Royal College of Surgeons of Edinburgh.

[With a Plate.]

\section{Class ANNELIDES *.}

Vers a sans rouge, Cuv. Règ. Anim. iii. 182.

Annelides, Lam. Anim. s. Vert. v. 274; 2nde edit. v. 499 . Flem. Phil. Zool. ii. 599. Latr. Fam. Nat. 235. Aud. et M, Edw. Hist. Nat. du Litt. de la France, ii. 1. Stark, Elem. ii. 127.

Vermes, Leach in Suppl. Encyclop. Brit. i. 450.

Annelina, M.Edwards in Cyclop. Anat. and Phys. i. 164. Roget,

Bridgew. Treat. i. 269. Grant; Outl. Comp. Anat. 31. Jones,

Anim. Kingd. 188-223. Griflith's Cuvier, xiii. 6.

Annulata, MacLeay in Ann. Nat. Hist. iv. 385.

Order 1. APODA.

(Macleay in Ann. Nat. Hist. iv. 385.)

Tribe Nemertina.

(MacLeay in Ann. Nat. Hist. iv. 385.)

Family Gondusidat.

Les Dragonneaux, Cuv. Règ. Anim. iii. 217.-Enterobranchia filiformia, Latr. Fam. Nat. 246.

Gordius.

Gordius, Linn. Syst. 1075. Lam. Anim. s. Vert. iii. 219 ; 2nde edit. iii. 671. Flem. Phil. Zool. ii. 605. Schweigg. Handb. 590.

1. G. aquaticus.

Seta aquatica, Merr. Pin. 207.

Gordius aquaticus, Linn. Syst. 1075. Turt. Gmel. iv. 57. Turt. Brit. Faun. 130. Stew. Elem. ii. 353. Penn. Brit. Zool. iv. 73. Flem. Phil. Zool. ii. 605. Baird in Trans. Berw. Nat. Club, i. 23.

* The true limits of this elass were first indicated by Pallas in his 'Miscellanea Zoologica," p. 74, Lugd. Bat. 1778. Lamarck conferred upon it the name Annelides in 1812.

t This family should be referred to the class Entozoa. See Reports on Zoolngy and Botany, translated for the Ray Society, p. 292.

A worm, presumed to be identical with the Mermis nigrescens of Dujardin, has been found in great abundance at Fairford in Gloucestershire. An account of it will be soon published, we have reason to believe, by the Rev. L. Jenyns.

Ann.\& Mag. N. Hist. Vol. xvi. Suppl. 
Templeton in Loud. Mag. N. Hist. ix. 236. Drummond in ibid. ix. 241. Johnston in ibid. ix. 355. fig. 52. Encyclop. Brit. xi, 226.

2. G. argillaceus.

Gordius argillaceus, Linn. Syst. 1075. Turt. Gmel. iv. 57. Turt. Brit. Faun. 130. Stew. Elem. ii. 353. Penn. Brit. Zool. iv. 73. Flem. Phil. Zool. ii. 605.

Obs. Dr. Fleming says this "is only a variety" of the preceding. I know of no description of it taken from British specimens. Species of Filarice have been frequently described as Gordii.

\section{Family Pranariada.}

Des Planaires, Cuv. Règ. Anim. iii. 266.-Mollusca Parenchymata, Swainson, Malacology, 35.

Subfamily Linina.

Borlasia.

Borlasia, eubgenus, Johnston in Mag. Zool, and Bot. i. 536.

1. B. ruffrons.

Nemertes B. rufifrons, Johnston in Mag. Zool. and Bot. i.538. pl. 18. fig. 5 .

2. B. purpurea.

Nemertes B. purpurea, Johnston in lib. cit. i. 537. pl. 18. fig. 3.

3. B. alba.

Borlasia? alba, W. Thompson's Report, p. 271 ; and in Ann. Nat. Hist. xv. 320 .

4. B. octoculata.

Nemertes B. octoculata, Johnston in lib. cit. i. 537. pl. 18. fig. 2.

Planaria octoculata, Johnston in Zool. Journ. iv. 56 .

5. B. olivacea.

Nemertes B. olivacea, Johnston in lib. cit. i. 536. pl. 18. fig. 1.

Planaria bioculata, Johnston in Zool. Journ. iv. 56.

6. B. ? fliformis. Plate XV. fig. $1, a, b$.

Planaria filiformis, Johnston in Zool. Journ. iv. 56.

Desc. Body very slender, elongate, abont the thickness of common sewing thread, of a uniform white or yellowish white colour, soft, smooth, contractile, thickest in front and subcylindrical, tapered insensibly backwards and flattened : mouth terminal, somotimes protruded like a papilla: eyes none: anus terminal. Under a magnifier the sides appear minutely crenulate: the skin is transparent, and the centre dusky from a straight intestine which runs from one extremity to the other, and has on each side of it a series of oblong cells so closely approximated that they might seem almost to be formed by the convolutions of a single vessel.

When lying at ease and contracted, this worm is from 1 to 2 inches in length, but it can, and does often voluntarily, extend itself until it is upwards of 6 inches, when it becomes a mere thread-like white line. In this state, the anterior extremity, from being obtuse, is thrust out into a needle-like point. It lives under stones, in muddy places, between tide-marks. 

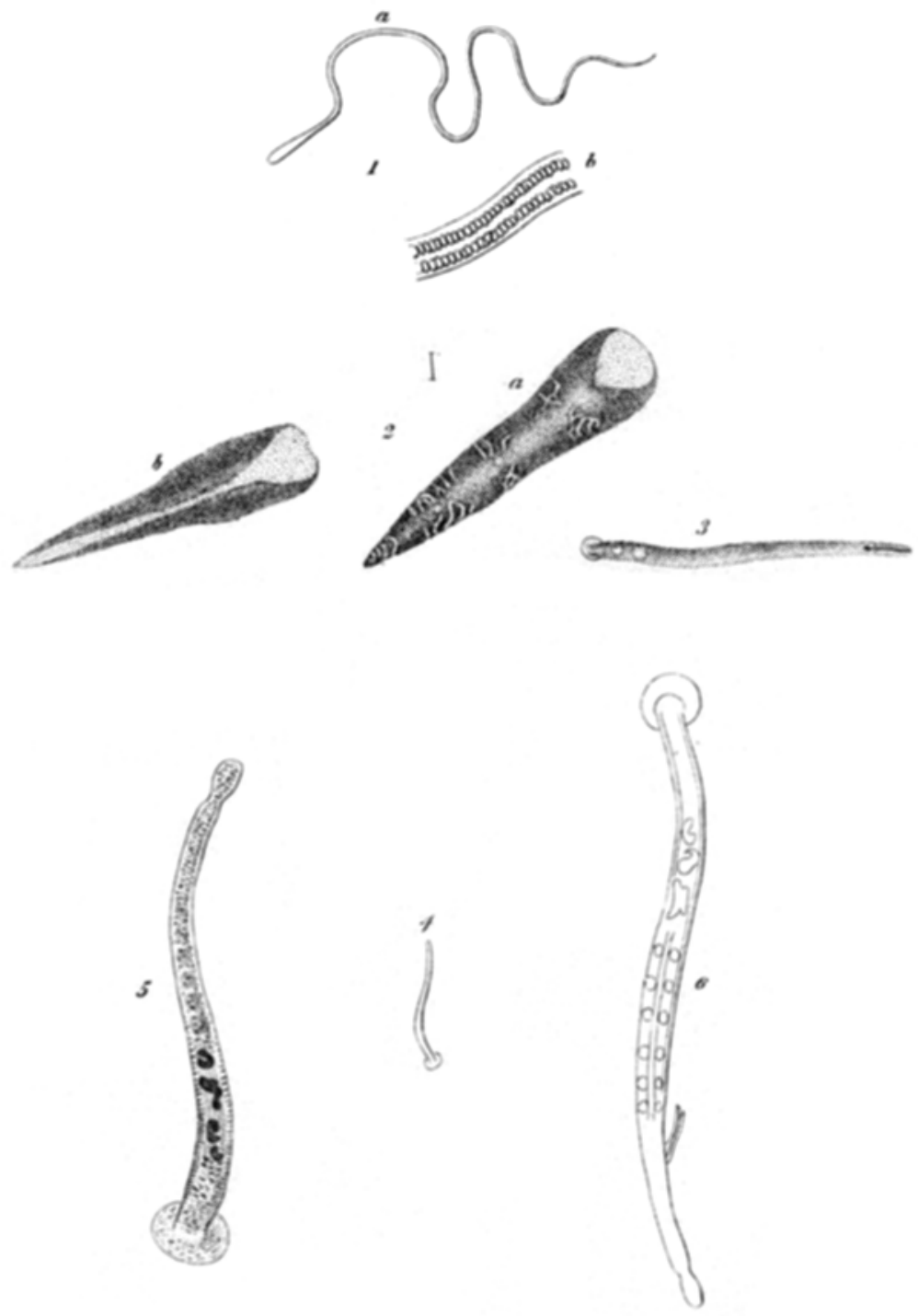
7. B.? flaccida.

Planaria flaccida, Johnston in Zool. Journ. iii. 488.

Obs. Not Planaria flaccida of Muller.

8. B. ? unicolor.

Planaria unicolor, Johnston in Kool. Journ. iii. 488.

\section{Lineus.}

Lineus, Sowerby, "Brit. Misc. tab. 8." Flem. Phil. Zool. ii, 605.Borlasia, Oken in Schweigg. Handb. 591.-Nemertes, Cuv. Règ. Anim. iii. 259.-Notospermus? Huschke in Edin. Journ. Nat. and Geogr. Sc. iii. 305.

1. L. longissimus.

Lineus longissimus, "Sowerby, Brit. Misc. tab. 8." Jameson in Wern. Mem. i. 557. 'Turt. Brit. Faun. 130.

Gordius marinus, Penn. Brit. Zool. iv. 74. Montagu in Linn. Trans. vii. 72. Davies in Linn. Trans. xi. 292.

Borlasia Angliæ, Schweigg. Handb. 591.

Nemertes Borlasii, Cuv. Règ. Anim. iii. 259. W. Thompson in Charlesw. Mag. ii. 21. Griffith's Cuv. xii. 468.

Borlasia longissimus, Templeton in Loud. Mag. N, Hist. ix. 236.

2. L. gracilis.

Nemertes, H. D. S. Goodsir in Ann. N. Hist. xv. 378. pl. 20. fig. 3.

Serpentaria.

Serpentaria, H. D. S. Goodsir in Ann. N. Hist. xv. 377.

1: S. fragilis.

Serpentaria, Goodsir in Ann. N. Hist. xv. 377. pl. 20. fig. 1, 2.

Meckelia.

Meckelia, Leuckart $=$ Carinella, Johnston.

1. M. trilineata.

Gordius annulatus, Montagu in Linn. Trans. vii. 74. Turt. Brit. Faun. 130. Penn. Brit. Zool. iv. 73.

Carinella trilineata, Johnston in Loud. Mag. N. Hist. vi. 232. fig. 24. W. Thompson in Ann. and Mag. N. Hist. vii. 482.

Obs. As this is distinct from the Meckelia annulata of Grube (Actin. \&c. p. 58. pl. 1. fig.7.), I have changed the specific name of Montagu.

\section{Prostoma.}

Prostoma, Dugès : v. Mag. Zool. and Bot. ii. 471.-Nemertes, Johnston in Mag. Zool. and Bot. i. 534.

1. P. gracilis.

Nemertes gracilis, Johnston in Mag. Zool. and Bot. i. 534. pl. 17. fig. 1. W. Thompson in Ann. and Mag. N. Hist. vii. 482.

2. P. lactiflorea.

Planaria lactiflorea, Johnston in Zool. Journ. iii. 489.

Nemertes lactiflorea, Johnston in Mag. Zool. and Bot. i. 535. pl. 17. fig. 2. W. Thompson in Ann, and Mag. N. Hist. vii. 482 . 
3. Pl.? rosea.

Planaria rosea, Mull. Zool. Dan. Prod.221. Zool. Dan. ii.31. tab.64. fig. 1, 2. W. Thompson in Ann. Nat. Hist. xv. 321.

Fasciola rosea, Mull. Verm. ii. 58.

4. P. quadrioculata.

Planaria quadrioculata, Johnston in Zool. Journ. iv. 56.

Nemertes quadrioculata, Johnston in Mag. Zool. and Bot. i. 535. pl. 17. fig. 4.

5. P. melanocephala.

Nemertes melanocephala, Johnstơn in Mag. Zool. and Bot. i. 535. pl. 17. fig. 5 .

6. P. pulchra.

Nemertes pulchra, Johnston in Mag. Zool. and Bot. i. 536. pl. 17. fig. 6.

7. P. armatum.

Prostoma armatum, Templeton in Loud. Mag. N. Hist. ix. 236. fig. 29. Edin. Journ. Nat. and Geogr. Sc. iii. 379.

Subfamily Planarina.

Planaria, Mell. Zool. Dan. Prod. xxviii.-Planariadæ, Flem. Phil. Zool. ii. 604.

\section{Planaria.}

Planaria, Lam, Anim. s. Vert. iii. 176. Flem. Phil. Zoul. ïi. 604. Schweigg. Handb. 593 (v. Edin. Journ. Nat. and Geogr. Sc. iii. 380).

1. P. vittata.

* Marine.

Planaria vittata, Montagu in Linn. Trans. xi. 25. tab. 5. fig. 3. W. Thompson in Ann. and Mag. Nat. Hist. v. 247.

2. P.atomata.

Planaria punctata, Mull. Zool. Dan. Prod. 2706.

- atomata, Mull. Zool. Dan. i. 37. tab. 32. fig. 3, 4. Turt. Gmel. iv. 65. Fleming in Edin. Phil. Journ. viii. 297.

3. P. cornuta.

Planaria cornuta, Mull. Zool. Dan. Prod. 2681. Zool. Dan. i. 37. tab. 32. fig. 5-7. Johnston in Loud. Mag. N. Hist. v. 344. fig. 79. W. Thompson in Ann. Nat. Hist. xv. 320.

4. P. tremellaris.

Planaria tremellaris, Mull. Zool. Dan, i, 36. tab. 32. fig. 1, 2. W. Thompson in Ann. and Mag. N. Hist. v. 247.

5. P. subauriculata.

Planaria subauriculata, Johnston in Loud. Mag. Nat. Hist. ix. 16. fig. 2.

6. P.flexilis.

Planaria flexilis, Dalyell on Planariæ, 5. pl. 1. fig. 1, 2. Johnston in Loud. Mag. N. Hist. ix. 17. 
7. P. stagnalis.

Fasciola stagnalis, Mull. Verm. i. ii. 53.

Planaria stagnalis, Mull. Zool. Dan. Prod. 221. Templeton in Loud. Mag. N. Hist. ix. 239.

8. P.macrocephala, oblonga, fusca, antice dilutior truncata, postice attenuata; oculis ocellisve nullis; long. corp. 2 lin. Pl. XV. fig. $2, a, b$.

Hab. Amongst Confervæ in Berwick bay.

Desc. Body brown, oblong, truncate before and paler coloured, narrowed posteriorly; no eyes nor eye-like points. The anterior extremity, in some positions, appears marked with a pale subcircular spot, while in others a light-coloured line runs down the centre, while the margins seem folded in so as to form almost a tube, as is well represented in our figure $b$. From this circumstance I conclude that the worm is thin with involute margins, but I never saw it spread itself out. There are two ventral pores, similar to those of the genuine Planarice.

I have named this species macrocephala because of the bluntness of its head, for the enlarged end is the anterior one, a fact which the mere examination of the figure would never resolve, since the part is distinguished by none of its usual appendages or organs: it has neither mouth, eyes, nor tentacula. But all unfurnished as it is, there is no species of its genus that I have met with that excels it in activity. Ever-restless, it glides along the surface of the vessel with great celerity, varies ite course with ease, and sometimes leaving the bottom, it swims in the bosom of the water, but with a tardier pace. With the obtuse anterior end, which is in constant motion and change, it feels the way, and pushing aside the floating particles that annoy it, it guides itself with as much safety, and appears tis careful of obstacles as if indeed eyes and feelers were sentinels in front.

9. P. Hirudo, lineari-elongata, fusca, antice puncta lineaque nigra signata ; maculis supra caudam orbicularem duabus pallidis. Long. corp. 4 lin. Pl. XV. fig. 3.

$H a b$. The sea amongst Confervæ.

Desc. Body linear, lengthened, brown, marked in front with a black point and line; two pale spots above the circular tail. Length 4 lines.

This little worm was also found in Berwick bay, and it is little less active than the preceding. It glides along in a continuous motion, and when inclined to turn or change the direction of its course, it forms the tail into a disc-like sucker to give itself the advantage of a fixed point. The body is of a uniform light brown colour excepting the two pale spots near the tail, and, when fully stretched, is of a narrow linear figure, slightly tapered in front. There is a small black dot about half a line behind the anterior end, and a dark line runs from it forward to the tip. These are not formed by an eye, or a cluster of eye-like points, but the line seems rather to be produced by some internal tube, and the dot by some harder, perhaps horny, substance.

This worm is of a doubtful genus. The blaek stria at the anterior end may perhaps indicate the existence of a proboscis; and the soft disc at the tail has some resemblance to the sucker of a leech. I retain it in the genus Planaria because the body is smooth without trace of wrinkles or rings, and because there are two obvious pore-like spots near the posterior extremity. The specific name, however, may serve to mark its affinity to some of the leech tribe.

\section{** Freshwater.}

10. P. nigra.

Planaria nigra, Mull. Zool. Dan. Prod. 221. Zool. Dan. iii. 48. 
tab. 109. fig. 3, 4. 'Turt. Brit. Faun. 128. Dalyell on Planariæ, 23. fig. 3-5 and 15 .

Planaria fusca, Penn. Brit. Zool. iv. 68.

Fasciola nigra, Mull. Verm. i. ï. 54.

Hirudo nigra, Kirby in Linn. Trans. ii. 317.

11. P. fusca.

Planaria fusca, Pallas, Templeton in Mag. N. Hist. ix. 239.

Fasciola torva, Mull. Verm. i. ii. 62.

Planaria torva, Mull. Zool. Dan. Prod. 2688. Zool. Dan. iii. 48. tab. 109. fig. 5, 6. Turt. Gmel. iv. 64 .

Planaria fusca, Turt. Gmel. iv. 64. Stew. Elem. ii. 355.

Planaria Arethusa, Dalyell on Planariæe, 85. fig. 11-14.

$O b s$. I have reduced $P l$. fusca and torva to one species on the authority of Dugès.

12. P. lactea.

Fasciola lactea, Mull. Verm. i. ii. 61 .

Planaria lactea, Mull. Zool. Dan. Prod. 2687. Zool. Dan. iii. 47. tab. 109. fig. 1, 2. Turt. Gmel.iv.64. Stew. Elem. ii. 355. Edin. Journ. Nat. and Geogr. Sc. iii. 242 and 243.

Hirudo alba, Kirby in Iinn. Trans. ii, 316.

Planaria alba, Turt. Brit. Faun. 129.

13. P.panniculata.

Planaria panniculata, Dalyell on Planariæ, 37. fig. 6, 7.

14. P. felina.

Planaria felina, Dalyell on Planariæ, 42. fig. 8.

\section{Dalyellia.}

Dalyellia, Flem. Phil. Zool. ii. 605.

1. D. graminea.

Hirudo viridis, Shaw in Linn. Trans. i. 92. Johnson on Med. Leech, 19. Planaria viridis, 'Turt. Brit. Faun. 129. Stew. Elem. ii. 355.

Planaria graminea, Dalyell on Planariæ, 114. fig. 16.

Obs. Distinct from the $P l$. viridis of Muller, but surely identical with his Pl. Helluo.

2. D. velox.

Planaria velox, Dalyell on Planariæ, 127. fig. 17.

3. D. edinensis.

Planaria edinensis, Dalyell on Planuriæ, 133. fig. 18.

Tribe Hintodna.

(Macleay in Ann. Nat. Hist. iv. 385.)

Les Hirudinées, Lam. Anim. s. Vert. v. 289. Flem. Phil. Zool, ij $603-4$.

Family Phyllinida. Udonella.

Udonella, Johnston in Loud. Mag. N. Hist. viii. 498. 
1. U. caligorum.

Udonella caligorum, Johnston in Loud. Mag. N. Hist. viii. 497. fig. 45. W. Thompson in Ann. and Mag. N. Hist. xv. 320.

Obs. Closely allied to, but distinct from, the Nitzschia elegans of

K. E. v. Baer in Nov. Act. Acad. Cæs. Nat. Cur. xiij. pl. 32. fig. 1-4. Malacobdella.

Malacobdella, Blainville in Cuv. Règ. Anim. iii. 217.

1. M. grossa.

Hirudo grossa, Mull. Zool. Dan. Prod, 2668. Zool. Dan. i. 21. tab. 21. fig. 1-5, copied in Encyclop. Méthod. pl. 52, fig. 6-10. Turt. Gmel. iv. 70.

Phylline grossa, Johnston in Loud. Mag. N. Hist. vii. 587. fig. 67.

Tristoma.

Tristoma, Cuv. Règ. Anim. iii. 265.

1. T. coccineum.

Tristoma coccineum, Cuv. Règ. Anim. iii. 265. pl. 15. fig. 10. Yarrell, Brit. Fish. 2nd edit. ii. 468. vign. Griffith's Cuv. xii. 473. pl. 9. fig. 10 .

Phylline coccinea, Schweigg. Handb. 474.

\section{Phylline.}

Phylline, Lam. Anim. s. Vert. v. 295 ; 2nde edit. v. 526.

1. Ph. Hippoglossi.

Lernææ species, Bast. Opuse. Subs, ii. 138. pl. 8. fig. 11.

Phylline Hippoglossi, Johnston in Ann. N. Hist. i. 431 . pl. 15. fig. 1-3. W. Thompson in Ann. and Mag. N. Hist. vii. 482.

Obs. The synonyms will be found in the 'Annals.' Muller's figures are copied in Encyclop. Méthod. pl.52. fig. 11-14.

Family Hroudinide.

Hirudo, Linn. Syst.1079.-Les Sangsues, Cuv. Règ. Anim. iii. 212.Hirudinea, Latr. Fam. Nat. 246.-Annelides suceuses, Aud. et M. Edwards, Litt. de la France, ii. 50.

\section{Erpobdella.}

Erpobdella, Blainville in Lam. Anim. s. Vert. v. 296 ; 2nde edit. v. 527.-Nephelis, Savigny: v. Lam. 2nde edit. v. 527.-Heluo, Oken: v. Schweigg. Handb. 593.

1. E.vulgaris.

Hirudo octoculata, Linn. Syst. 1079. Turt. Gmel.iv. 69. Turt. Brit. Faun. 129. Penn. Brit. Zool. iv. 71.

Hirudo vulgaris, Mull. Verm. i. ii. 40. Stew. Elem. ii. 356. Johnson on the Med. Leech, 33. Furth. Obs. 29, with a plate.

Erpobdella vulgaris, Lam. Anim. s.Vert. 2nde edit.v. 528. D. Chiaje, An. s. Vert. Nap. i. 49.

Nephelis rulgaris, Brightwell in Ann. and Mag. N. Hist. ix. 13. pl.1. fig. 9ـ14.

Nondescript Leech? Ure's Rutherglen, 236. 
2. E. tessulata.

Hirudo tessulata, Mull. Verm. i. ii. 45. Johnson on the Med. Leech, 33. Fleming in Wern. Mem. iii. 400.

Erpobdella tessulata, Flem. Phil. Zool. ii. 604.

Nephelis tessulata, Brightwell in Ann. and Mag. N. Hist. ix. 13. pl.1. fig. 15-17.

3. E. lineata.

Hirudo lineata, Mull.Verm. i. ii. 39. Johnson on the Med. Leech, 34. Fleming in Wern. Mem. iii. 400.

Erpobdella lineata, Flem. Phil, Zool. ii. 604.

\section{Glossopora.}

Glossiphonia, Johnson on the Med. Leech, 25.-Glossopora, Johnson, Furth.Obs. 48.-Clepsine, Savigny._-Glossobdeila, Blainville : see Lam. Anim. s. Vert. 2nde edit. v. 528.

1. G. complanata.

Hirudo complanata, Linn. Syst. 1079. Mull.Verm. i. ii. 47. Turt. Gmel. iv. 69. Turt. Brit, Faun. 129. Stew. Elem. ii. 357. Penn. Brit. Zool. iv. 72. Ure's Rutherglen, 233.

Hirudo crenata, Kirby, Linn. Trans. ii. (his description is copied in Johnson, Med. Leech, p. 21). Turt. Gmel. iv. 71. Penn. Brit. Zool. iv. 71. Turt. Brit. Faun. 129.

Clepsina crenata, Encyclop. Brit. xi. 226. pl. 276. fig. 13.

Glossiphonia tuberculata, Johnson on the Med. Leech, 25.

Glossopora tuberculata, Johnson's Ferth. Obs. 49. pl. 17. fig. 1-10. Stark, Elem. ii. 142. W. Thompson in Ann. and Mag. N. Hist. vii. 482.

Erpobdella complanata, Lam. Anim. s. Vert. 2nde edit. v. 528. Templeton in Loud. Mag. N. Hist. ix, 235.

Erpobdella crenata, Templeton in Loud. Mag. N. Hist. ix. 235.

Glossopora complanata, Flem. Phil. Zool. ii. 604.

Clepsina complanata, Brightwell in Ann. and Mag. N. Hist. ix. 14. pl. 1. fig. 18, 19.

2. G. bioculata.

Hirudo stagnalis, Linn. Syst. 1079. Turt. Gmel. iv. 69. Turt. Brit. Faun. 129. Penn. Brit. Zool. iv. 71.

Hirudo bioculata, Mull. Verm. i. ii. 41. Stew. Elem. ii. 357. Ure's Rutherglen, 234.

Glossiphonia perata, Johnson on the Med. Leech, 26.

Glossopora punctata, Jahnson's Furth. Obs. 50. pl. 17. fig. 11-13.

Erpobdella bioculata, Lam. Anim. s. Vert. 2nde edit. v. 528.

- stagnalis, Templeton in Loud. Mag. N. Hist. ix. 235.

Glossopora bioculata, Flem. Phil. Zool. ii. 604.

Clepsina stagnalis, Brightwell in Ann. and Mag. N. Hist. ix. 14.

3. G. ? circulans.

Hirudo circulans, Sowerby : see Johnson on Med. Leech, 27. Turt. Brit. Faun. 129. Penn. Brit. Zool. iv. 72.

4. G. ? hyalina.

Hirudo heteroclita. Jinn. Syst, 1080. Johnson on Med. Leech, 34 . 
Un ver plat et blanc, Tremb. Polyp. 147. pl. 7. fig. 7.

Hirudo hyalina, Mull. Verm. i. ii. 49. Stew. Elem. ii. 357.

Clepsina hyalina? Brightwell in Ann. and Mag. Nat. Hist. ix. 15. pl. 1. fig. 20.

Piscicola.

Piscicola, Lam. Anim. s. Vert. 2nde edit. v. 525.-Hæmocharis, $S a$ vigny. (See Ann. and Mag. N. Hist. ix. 11.)

1. P. geometra.

Hirudo geometra, Linn. Syst. 1080. Turt. Gmel. iv. 70. Turt. Brit. Faun. 129. Johnson on the Med. Leech, 35. Brightwell in Ann. and Mag. N. Hist. ix. 11. pl. 1. fig. 1-8.

Hirudo piscium, Mull. Verm. i. ii. 43. Penn. Brit. Zool. iv. 70. pl. 21. fig. 3. Stew. Elem. ii. 357.

Piscicola piscium, Lam. Anim. s.Vert. 2nde edit. v. 525. Stark, Elem. ii. 142 .

Piscicola geometra, Flem: Phil. Zool. ii. 604.

2. P. Perce.

Ichthyobdella Percæ, Templeton in Loud. Mag. N. Hist. ix. 236. fig. 28 .

3. P. marina. Plate XV. fig. 4-6.

Hab. Parasitical on the Aspidophorus cataphractus.

Desc. Body one inch in length and a line in diameter, slightly tapered forwards, terminated at each end with a plain circular sucker, of a uniform chestnut-brown colour, or red and mottled (for the colour varies according to the intestinal contents), smooth, rather soft and compressed; the margins minutely crenulate under a magnifier.

Fig. 4. P. marina of the natural size. 5. The same magnified. 6. An outline figure drawn from an individual which lad the genital organs extruded.

Obs. Mr. W. Thompson has, in his Catalogue of the Irish Worms, a Piscicola marina (Rep. p. 272), but no description of it has been published.

\section{Pontobdella.}

Pontobdella, Leach in Suppl. Encyclop. Brit. i. 451. Lam. Anim.s. Vert. v. 293.

1. P. muricata.

Hirudo piscium, Bast. Opusc. Subs. i. 82. tab. 10. fig. 2.

Hirudo muricata, Linn. Syst. 1080. Turt. Gmel. iv. 71. Turt. Brit. Faun. 130. Stew. Elem. ii. 357. Penn. Brit. Zool. iv. 71. pl. 21. fig. 4. Johnson on the Med. Leech, 38. Dalyell in Edin. New Phil. Journ. 1827, p. 391. Grant in Edin. Journ. of Science, no. 14.

Hirudo verrucosa, Fleming in Wern. Mem. ii. 245. Johnson on the Med. Leech, 39.

Pontobdella muricata, Lam. Anim. s.Vert. v. 293 ; 2nde edit. v. 524.

Stark, Elem. ii. 142. Templeton in Loud. Mag. N. Hist. ix. 236. Albione muricata, D. Chiaje, An. s. Vert. Nap. i. 49. 
2. P. spinulosa.

Pontobdella spinulosa, Leach, Zool. Misc. ii. tab. 65 ; and in Suppl. Encyclop. Brit. i. 451. pl. 26. Lam. Anim. s. Vert. v. 294; 2nde edit. v. 525. Stark, Elem. ii. 142.

Obs. I doubt the distinctness of this from the preceding.

\section{Hamopsis.}

Hæmopsis, Savigny in Lam. Anim. s.Vert. 2nde edit. v. 520. Encyclop. Brit. xi. 226. pl. 176. fig. 15.

1. H. vorax.

'The Horse-Leech, Sibb. Scot. ii. 3, 34. Quekett in the Zoologist, i. 17 and 88 .

Hirudo Sanguisuga, Merr. Pin. 207. Linn. Syst. 1079. Turt. Gmel. iv. 68. Turt. Brit. Faun. 129. Stew. Elem. ii. 356. Penn. Brit. Zool. iv. 70. Johnson on the Med. Leech, 30. Mull. Verm. i. ii. 38. Stark, Elem. ii. 356. Templeton in Loud. Mag. N. Hist. ix. 235. Ephem. Acad. Leop. c. viii. 346. tab. 5. fig. 4. D. Chiaje, An. s. Vert. Nap. i. 48.

Hirudo sanguisorba, Lam. Anim. s. Vert. 2nde edit. v. 521.

Hirudo vorax, Johnson on the Med. Leech, 62.

Hæmopsis sanguisorba, Brightwell in Ann. and Mag. N. Hist. ix. 12. Encyclop. Brit. xi. 226.

2. H. nigra.

Hirudo nigra, Johnson on the Med. Leech, 32.

Obs. In the last edit. of Lamarck this is considered as identical with the preceding.

\section{Hirudo.}

Sanguisuga, Savigny in Lam. Anim. s.Vert. 2nde edit. v. 520. Encyclop. Brit. xi. 225.

1. H. medicinalis.

Hirudo medicinalis, Linn. Syst. 1079. Mull. Verm. i. ii. 37. Turt. Gmel. iv. 68. 'Turt. Brit. Fann. 129. Stew. Elem. ii. 356. Penn. Brit. Zool. iv. 69. Stark, Elem, ii. 143. Leach in Suppl. Encyclop. Brit. i. 451 . pl. 26. Johnson on the Med. Leech, 29. Lam. Anim. s. Vert. 2nde edit. v. 520. Home, Comp. Anat.iv. pl. 39. fig. 3 ; ibid. ii. 70 . Ephem. Acad. Leop. cent. viii. 338. tab. 5. fig. 1, 2, 3. D. Chiaje, An. s. Vert. Nap. i. 47.

Sanguisuga medicinalis, Brightwell in Ann. and Mag. N. Hist. ix. 13. Encyclop. Brit, xi. 225. pl. 176. fig. 14.

\section{Tribe Lumbricina.}

(Macleay in Ann. Nat. Hist. iv. 385.)

Annelides abranches setigères, Cuv. Règ. Anim. iii. 209.-Lombricini, Latr.Fam.Nat.246.-Annelides terricoles, Aud. et M. Edwards, Litt. de la France, ii. 50; and in Lam. Anim. s. Vert. 2nde edit. v. 513. 
Family Naïdes.

Les Nä̈des, Cuv. Règ. Anim. iii. 211.-Vers hispides, Lam. Anim. s. Vert. iii. 221. (See Rep. trans. by Ray Society, p. 282.)

\section{Nais.}

Nais, Mull. Zool. Dan. Prod. xxviii. Verm. i. ii. 20. Turt. Gmel. iv. 91. Lam. Anim. s. Vert. iii. 222. Leach in Suppl. Encyclop. Brit. i. 451.

1. N. vermicularis.

Nais vermicularis, Mull. Verm. i. ii. 20. Lam. Anim. s. Vert. 2nde edit. iii. 674. Templeton in Loud. Mag. N. Hist. ix. 235. (Vide Zool. Journ. v. 380.)

2. N. serpentina.

Nais serpentina, Mull. Verm. i. ii. 20. Mull. Wurm. 84. tab. 4. fig. 1-4. Turt. Gmel. iv. 91. 'Turt. Brit. Faun. 137. Stew. Elem. i. 390. Penn. Brit. Zool. iv. 97. Templeton in Loud. Mag. Nat. Hist. vii. 130. fig. 26 ; and ix. 235.

3. N. Lurco.

The Lurco or Glutton, a diaphanous species of Nais, Pritchard, Micros. Cabinet, 78. pl. 8. fig. 1 .

4. N. digitata.

Nais digitata, Mull. Verm. i. ii. 22. Wurm. 90. tab. 5. fig. 1-4. Turt. Gmel. iv. 91. Turt. Brit. Faun. 137. Stew. Elem. i. 391. Penn. Brit. Zool, iv, 98. Bose, Vers, i. 239.

\section{Stylaria.}

Stylaria, Lam. Anim. s. Vert. iii. 223.

1. S. lacustris.

Mille-pied à dard, Trembl. Polyp. 144. pl. 6. fig. 1 .

Nereis lacustris, Linn. Syst. 1085.

Nais proboscidea, Mull. Zool. Dan. Prod. 2649. Wurm. 14. tab. 1. fig. 1-4. Verm. i. ii. 21. Turt. Gmel. iv. 91. Turt. Brit. Faun. 137. Stew. Elem. i. 390. Penn. Brit. Zool, iv. 97. Baker, Employm. Micros. 317. pl. 12. fig. 24.

Stylaria paludosa, Lam. Anim. s. Vert. 2nde edit. iii. 675.

- lacustris, Templeton in Loud. Mag. N. Hist. ix. 235.

\section{Tubifex.}

Tubifex, Lam. Anim. s. Vert. iii. 224. Schweigg. Handb. 590.

1. T. rivulorum.

Vermes minimi rubri aquam stagnalem colore sanguineo inficientes, unde vulgus dira portendit, Merr. Pin. 207.

Vers qu'on trouvent souvent rassemblés en grande quantité au fond de l'eau, Trembl. Polyp. 98, 99 and 147. pl. 7. fig. 2.

Small red Water-worms found plentifully in the mud of the river Thames, Baker, Polype, 62.

Lumbricus tubifex, Mull. Zool. Dan. Prod. 2605. Wurm. 62. Verm. i. ii. 27. Zool. Dan. iii. 4. tab. 84. Bosc, Vers, i. 255. 
Tubifex rivulorum, Iam. Anim. s. Vert. 2nde edit. iii. 676. Templeton in Loud. Mag. N. Hist. ix. 235.

\section{Family Lumbarcinds.}

Lumbricus, Linn. Syst. 1076.-Les Lombrics, Cuv. Règ. Anim. iii. 209.-Les Echiurées, Lam. Anim. s. Vert. v. 297.

\section{Lumbricus.}

Lumbricus, Cuv. Règ. Anim. iii. 209. Lam. Anim. s. Vert. v. 298. Schweigg. Handb. 590. Leach in Suppl. Encyclop. Brit. i. 451.

* Marine.

1. L. lineatus.

Faden-Wurm, Mull. Wurm. 118. dritte taf. fig. 4, 5 .

Lumbricus lineatus, Mull. Verm. i. ii. 29. Johnston in Zool. Journ. iii. 329 ; and in Loud. Mag. Nat. Hist. viii. 259. fig. 24.

2. L. capitatus.

Lumbricus capitatus, Johnston in Loud. Mag. Nat. Hist. viii. 258. fig. 23.

Lumbricus littoralis, Johnston in Zool. Journ. iii. 328.

3. L. minutus.

Lumbricus minutus, Fabric. Faun. Groenl. 281. pl. 1. fig.4. Lam. Anim. s. Vert. 2nde edit. v. 533. Johnston in Zool. Journ. iii. 328.

4. L. rufescens.

Lumbricus rufescens, Johnston in Zool. Journ. iii. 327.

5. L. vermicularis.

$$
\text { ** Subaquatic. }
$$

Lumbricus vermicularis, Mull. Verm. i. ii. 26. Flem. PhiT. Zool. ii. 603. Johnston in Zool. Journ. iv. 421.

6. L. variegatus.

Lumbricus variegatus, Mull.Verm. i. ii. 26. Flem. Phil. Zool. ii. 603. Johnston in Zool. Journ. iii. 326. Bosc, Vers, i. 255.

7. L. pellucidus.

Lumbricus ? Clitellio? pellucida, Templeton in Loud. Mag. N. Hist. vii. 131. fig. 27.

Clitelis minutus, Templeton in Loud. Mag. N. Hist. ix. 235.

Obs. The Lumbricus pellucidus (Johnston in Zool. Journ. iii. 327. comp. with iv. 421) is probably a larva.

8. L. omilurus.

*** Terrestrial.

Lumbricus omilurus, Templeton in Loud. Mag. N. Hist. ix. 235.

9. L. lividus.

Lumbricus lividus, Templeton in Loud. Mag. N. Hist. ix. 235.

10. L. gordianus.

Lumbricus gordianus, Templeton in Loud. Mag. N. Hist. ix. 235. 
11. L. xanthurus.

Lumbricus xanthurus, Templeton in Loud. Mag. N. Hist. ix. 235.

12. L. annularis.

Lumbricus annularis, Templeton in Loud. Mag. N. Hist. ix. 234.

13. L. terrestris.

Earth-Worm, Sibb. Scot. ii. 3, 33.

Vermes terrestres majores, Merr. Pin. 206.

Lumbricus terrestris, Willis, Oper. Omn. ii. 18. tab. 4. Linn. Syst. 1076. exclus. $\gamma$. Mull. Verm. i. ii. 24. Turt. Gmel. iv.58. Turt. Brit. Faun. 128. Home, Comp. Anat. iv. pl. 40. fig. 4, and pl.145, 146, 147, 148, 149. Penn. Brit. Zool. iv. 63. pl. 20. fig. 1, and fig. 2. var. minor. Stew. Elem. ii. 354. Stark, Elem. ii. 141. Leach in Suppl. Encyclop. Brit. i. 451. pl. 26. Templeton in Loud. Mag. N. Hist. ix. 234.

Common Earth-Worm, White's Selborne, ii. 14 and 279. Edin. Phil. Journ. iii. 412. Loudon's Suburb. Hortic. 94. Gray in Ann. Nat. Hist. ii. 479.

"De Lumbrici terrestris historia naturali necnon Anatomia tractatus. Auctore Carolo-F.-A. Morren." Bruxellis, 1829. In reference to this excellent work, see Edin. Journ. Nat. and Geogr. Sc. iii. 375 ; Loud. Mag. Nat. Hist. ix. 240.

\section{Cirratulus.}

Cirratulus, Lam. Anim. s. Yert. v. 300. Aud. and M. Edwards, Litt. de la France, ii. 268. Johnston in Mag. Zool. and Bot. ii. 71.

1. C. medusa.

Cirratulus medusa, Johnston in Mag. Zool. and Bot. ii. 71. pl. 3. fig. 7-12.

2. C. tentaculatus.

Terebella tentaculata, Montagu in Linn. Trans. ix. 110. pl. 6. fig. 2, copied in Encyclop: Brit. xi. pl. 276. fig, 4. Penn. Brit. Zool. iv. 111.

Cirrhatula tentaculata, Templeton in Loud. Mag. N. Hist. ix. 234.

Cirrhatulus tentaculatus, Mag. Zool, and Bot. ii. 73 ; and Encyclop. Brit. xi. 221.

\section{Trophonia.}

Trophonia, Johnston in Ann. Nat. Hist. iv. 371.

1. T. Goodsirii.

Trophonia Goodsirii, Johnston in Ann. Nat. Hist. iv. 371. pl. 11. fig. $1-10$.

\section{Travisia.}

Travisia, Johnston in Ann. Nat. Hist. iv. 373.

1. T. Forbesii.

Travisia Forbesii, Johnston in Ann. Nat. Hist. iv. 373. pl. 11. fig. 11-18. 


\section{Order II. POLYPODA.}

(MacLeay in Ann. Nat. Hist. iv. 385.)

Gymnobranchia, Leach in Suppl. Encyclop. Brit. 450.

Tribe Serpulina.

(MacLeay in Ann. Nat. Hist. iv. 385.)

Les Tubicoles, Cuv. Règ. Anim. iii. 189,-Annelides sédentaires, Lam. Anim. s.Vert.v. 332.-Cephalobranchia, Latr. Fam. Nat. 241. (See Pallas, Misc. Zool. 114.)

\section{Family Auricomides.}

Les Amphitrites, Cuv. Règ. Anim. iii. 194.

\section{Pectinaria.}

Pectinaria, Lam. Anim. s. Vert. v. 348.-Cistena, Leach in Suppl. Encyclop. Brit. i. 452.

1. P. belgica.

Nereis cylindraria belgica, Pallas, Misc. Zool. 122. pl. 9. fig. 3-13.

Sabella granulata, Linn. Syst. 1268. Mont. Test. Brit.544. Stew. Elem. ii. 423. Penn. Brit. Zool. iv. 369. Turt. Brit. Faun. 202.

Sabella tubiformis ? Penn. Brit. Zool. iv. 372. pl. 95. fig. 2.

Nereis pectinata, Sowerby. Turt. Br. Frun. 134.

Sabella belgica, Turt. Gmel. iv. 611 .

Amphitrite auricoma, Mull. Zool. Dan. Prod. 2622. Zool. Dan. i. 26. pl. 26. fig. 1-6. Fabric. Faun. Groenl. 289. Cuv. Règ. Anim. iii. 195. Penn. Brit. Zool. iv. 91. Stew. Elem. i. 389. pl. 12. fig. 1, 2 .

Pectinaria belgica, Lam. Anim. s.Vert. 2nde edit. v. 602. 'Templeton in Loud. Mag. Nat. Hist. ix. 234.

Cistena Pallasii, Leach in Suppl. Encyclop. Brit. i. 452. pl. 26.

2. P. ? arenaria.

Sabella arenaria, Mont. Test. Brit. 552. Penn. Brit. Zool, iv, 371. Turt. Brit. Faun. 203.

3. $P$.? subcylindrica.

Sabella subcylindrica, Mont. Test. Brit. 552. Turt. Brit. Faun. 203. Penn. Br. Zool. iv. 371.

4. P. ? setiformis.

Sabella setiformis, Mont. Test. Brit. 553. 'Turt. Br. Faun. 203. Penn. Br. Zool. iv. 371.

\section{Sabellaria.}

Sabellaria, Lam. Anim. s. Vert. v. 350.

1. S. alveolata.

Tubularia arenosa anglica, Ellis, Corall. 90. pl. 36.

Sabella alveolata, Linn. Syst. 1268.

Amphitrite alveolata, Cuv. Règ. Anim. iii. 195.

Sabellaria alveolata, Lam. Anim. s.Vert. 2nde edit. v. 605. Stark, Elem. ii. 133. Templeton in Loud. Mag. N. Hist. ix. 234. 
2. S. crassissima*.

Sabella alveolata, Penn. Brit. Zool. iv. 368. pl. 95. fig. sup. Mont. Test. Brit. 540.

Sabellaria crassissima, Lam. Anim, s.Vert. 2nde edit. v. 605. Templeton in Loud. Mag. ix. 234.

\section{Flemingia plumosa.}

\section{Flemingia.}

Amphitrite plumosa, Mull. Zool. Dan. Prod. 2621. Fabr. Faun. Groenl. 288. Mull. Zool. Dan. ii. 16. tab. 90. fig. 1, 2. Turt. Gmel. iv. 83 .

Flemingia muricata, Johnston in Berw. Trans. i. 15 (1832).

Obs. I do not know where to place this singular worm. My specimen was sent to the British Museum.

Desc. Body an inch long, cylindrical, tapered at the posterior end, of a dirty light brown colour, annulose, rough with numerous papillæ and hairy. No head nor eyes. Tentacula projected from the anterior extremity, completely retractile, of two kinds ; for two of them are filiform, equal, yellowish and fleshy; and eight of them-placed above and within the first-are setaceous, bristle-like, and of a bright grass-green colour. There are likewise about the anterior end numerous long bristles in indistinct fascicles, which, when magnified, appear distinctly jointed similar to a Conferva. Along each side a row of about sixty feet, one pair to each ring. The feet are papillary, each bearing about four unequal bristles, not retractile : those of the two or three anterior pairs are longer than the others. Anus terminal, simple. The animal can inject a green-coloured liquid into a central vessel of the fleshy tentacula.

One specimen found amongst the entangled roots of Laminaria digitata.

\section{Family Teregendom.}

\section{Terebella.}

Terebella, Cuv. Règ. Anim. iii. 193. Lam. Anim. s. Vert. v. 353.

I. T. conchilega.

Nereis conchilega, Pall. Misc. Zool, 131. tab. 9. fig. 14-22. "Penn. Brit. Zool. iv. 47."

Terebella conchilega, Turt. Gmel. iv. 84. Turt. Brit. Faun. 132. Penn. Br. Zool. iv. 112. Lam. Anim. s. Vert. 2nde edit. v. 607. Edin. Journ. Nat. and Geogr. Sc. iii. 244. Templeton in Loud. Mag. N. Hist. ix. 233.

Sabella rudis, Penn. Br. Zool. iv. 368.

- conchilega, Mont. Test. Brit. 547. Stew. Elem. ii. 423. Turt. Brit. Faun. 203.

2. T. chrysodon.

Sabella chrysodon, Mont. Test. Brit. 546. Penn. Brit. Zool. iv. 370. Turt. Brit. Faun. 202. Hogg's Stockton, 29.

* Sabella alveolata-Turt. Gmel, iv. 610,-Turt. Brit. Faun. 202,-Stew. Elem. ii. 423.--embraces both species. 
3. T. lumbricalis.

Sabella lumbricalis, Mont. Test. Brit. 549. Turt. Brit. Faun. 203. Stew. Elem. ii. 423. Penn. Brit. Zool. iv. 370.

4. T. cristata.

Amphitrite cristata, Mull. Zool. Dan. Prod. 2620. Zool. Dan. ii. 40. tab. 70. fig. 1-4. Jameson in Wern. Mem. i. 558. Stew. Elem. i. 389. Penn. Brit. Zool. iv. 91.

Terebella cristata, Lam. Anim. s.Vert. 2nde edit. v. 607. Templeton in Loud. Mag. N. Hist. ix. 233.

5. T. cirrhata.

Terebella cirrhata, Montagu in Linn. Trans. xii. 342. tab. 12. fig. 1. Sabella cirrata, Mont. Test. Brit. 550. Penn. Brit. Zool. iv. 370. Turt. Brit. Faun. 203.

6. T. nebulosa.

Terebella nebulosa, Montagu in Linn. 'Trans. xii. 343. tab. 12. fig. 2.

7. T. constrictor.

Terebella constrictor, Montagu in Linn. Trans. xii. 343. tab. 13. fig. 1.

8. T. venustula.

Terebella venustula, Montagu in Linn. Trans. xii. 344. tab. 13. fig. 2.

9. T. gigantea.

Terebella gigantea, Montagu in Linn. Trans, xii. 341. tab. 11.

Family Sabeltide.

Les Sabelles, Cuv. Règ. Anim. iii. 191.

\section{Othonia.}

Fabricia, Blainville.-Othonia, Johnston in Loud. Mag. N. Hist. viii. 183.

1. O. Fabricii.

Tubularia Fabricia, Mull. Zool. Dan. Prod. 254. Fabt. Faun. Groenl. 440. fig. 12. Turt. Gmel. iv. 668.

Tubularia stellaris, Sars in Ann. des Sc. Nat. 2nde sér. vii. 274.

Othonia Fabricii, Johnston in Loud. Mag. N. Hist. viii. 181. fig. 19.

Sabella.

Sabella, Cuv. Règ. Anim. iii. 191. Schweigg. Handb. 599.-Amphitrite, Lam. Anim. s. Vert. v. 354.

1. S. amona.

Sabella amœena, Johnston in Loud. Mag. N. Hist. vi. 406. fig. 53.

2. S. reniformis.

Nieren-formige Amphitrite, Mull. Wurm. 194. tab. 16.

Tubularia penicillus, Mull. Zool.Dan. Prod. 3063. Zool. Dan.tab.89. 
fig. 1, 2. Fabric. Faun. Groenl. 438. Fleming in Edin. Phil. Journ. viii. 295.

Amphitrite reniformis, Turt. Gmel. iv. 82.

— penicillus, Lam. Anim. s. Vert.v. 356; 2nde edit. v. 610.

3. S. infundibulum.

Amphitrite infundibulum, Montagu in Linn. Trans. ix. 109. tab. 8. Penn. Br. Zool. iv. 89. Lam. Anim. s. Vert. 2nde edit. v. 611.

4. S. vesiculosa.

Amphitrite vesiculosa, Montagu in Linn. Trans.xi. 19. tab. 5. fig. 1 . Stark, Elem. ii. 133. Lam. Anim. s. Vert. 2nde edit. v. 610.

5. S. Penicillas.

Scolopendra major tubularia, Bast. Opusc. Subs. i. ii. 77. tab. 9. fig. I. Sabella penicillus, Linn. Syst. 1269. Mont. Test. Brit. 541. Penn. Brit. Zool. iv. 369.

Nereis lutaria, Pall. Misc. Zool. 116. tab. 10. fig. 1.

Amphitrite ventilabrum, var. 2, Turt. Gmel. iv. 82. Jameson in Wern. Mem. i. 558. Turt. Brit. Faun. 136. Stew. Elem. i. 389. Penn. Brit. Zool. iv. 90. Dalyell in Edin. New Phil. Journ. xvii. 415. Lam. Anim.s. Vert. 2nde edit. v. 610.

6. S. carnea.

A Sabella resembling the Penicillus, Mont. Test. Brit. 544.

7. S. tubularia.

\section{***}

Serpula tubularia, Mont. Test. Brit. 513, and Suppl. 171. Turt. Brit. Faun. 202. Fleming in Edin. Encyclop. vii. 67. pl. 204. fig. 9. Penn. Br. Zool. iv. 362. Dillw. Rec. Sh. 1083. Fleming in Edin. Phil. Journ. xii. 243. Johnston in Loud. Mag. N. Hist. vii. 126. fig. 23. Berkeley in ibid. vii. 421. Laskey in Wern. Mem. i. 413. Brown, Illust. pl. 2. fig. 9, 10. Turt. Conch. Dict. 154. fig. 84.

Serpula arundo, Turt. Conch. Dict. 155. Berkeley in Zool. Journ. iii. 229.

Sabella tubularia, Berkeley in Zool. Journ. v. 426.

8. Serpula Cordineri.

Serpula Cordineri, Fleming in Edin. Phil. Journ. xii. 244.

*****

9. S. volutacornis.

Amphitrite volutacomis, Montagu in Linn. Trans. vii. 80. tab. 7. fig. 10. Lam. Anim. s. Vert. 2nde edit. v. 61l. Turt. Brit. Faun. 136. Penn. Brit. Zool. iv. 89. Stark, Elem. ii. 133. Leach in Suppl. Encyclop. Brit. i. 452. pl. 26.

Sabella curta, Mont. Test. Brit. 554. Penn. Br. Zool. iv. 372. Turt. Brit. Faun. 203.

Sabella compressa, Mont. Test. Brit. 555. Turt, Br. Faun. 203. Penn. Brit. Zool. iv. 372.

Ann. \& Mag. N. Hist. Vol. xvi. Suppl. $2 \mathrm{~K}$ 
Amplitrite rosea, Sowerby. Penn. Brit. Zool. iv. 90. Turt. Br. Faun. 136.

\section{Family Serpulide.}

Serpula, Linn. Syst. 1264.-Les Serpules, Cuv. Règ. Anim. iii. 190. -Les Serpulíes, Lam. Anim. s. Vert. v. 357.-Serpulcea, Latr. Faun. Nat. 242. (See Philippi in Ann. \& Mag. N. Hist. xiv. p. 153.)

Obs. In this family (Serpulida) I incline to arrange all the Dentalia included in the genus Brochus of Brown, the Cacalium of Fleming, the microscopic or minute Orthocera, and the greater part of our multilocular shells arranged in the genus Nautilus.

\section{Lobatula.}

Lohatula, Flem. Phil. Zool. ii. 602. Flem. Brit. Anim. 232.

1. L. vulgaris.

Nautilus lobatulus, Turt. Gmel. iv. 307. Turt. Brit. Faun. 166. Penn. Br. Zool. iv. 248. Dillw. Rec. Sh. 343. Turt. Conch. Dict. 120. Fleming in Encyclop. Edin. vii. 85.

Serpula lobata, Mont. Test. Brit. 515 , and Suppl. 160. Lowe in Zool. Journ. iii. 78.

Lobatula farcta, Flem. Phil. Zool. ii. 602.

Lobatula vulgaris, Flem. Br. Anim. 232.

Discorbis vesicularis, Clark in Zool. Journ. iii. 341.

Truncatulina tuberculata, Morris, Cat. Br. Foss. p. 64.

2. L. concamerata.

Serpula concamerata, Mont. Test. Br. Suppl. 160. Penn. Br. Zool. iv. 366. Lowe in Zool. Journ. iii. 79.

Nautilus concameratus, Fleming in Edin. Encycl. vii. 85.

Lobatula concamerata, Flem. Brit. Anim. 233.

\section{Spirorbis.}

Fleming in Edin. Encyclop. vii. 68. Lam. Anim. s. Vert. v. 358. Leach in Suppl. Encyclop. Brit. i. 452. Schweigg. Handb. 60I.

$$
\text { *Spirorbis, Flem. Phil. Zool. ii. } 602 .
$$

1. S. communis.

Vermiculus exiguus albus nautiloides algæ fere adherens, List. Conch. 553. 5.

Planorbis minimus, Petiv. Gazophy. tab. 35. fig. 8.

Serpula spirorbis, Linn. Syst. 1\%65. Da Costa, Brit. Cunch. 22. tah, 2. fig. 11. Fabric. Faun. Groenl. 377. Mull. Zool. Dan. iii. 8. tab. 86. fig. 1-6. Turt. Gmel. iv. 603. Mont. Test. Brit. 498. Laskey in Wern. Mem. i. 412. Brown in ibid. ii. 534. Penn. Brit. Zool. iv. 358. pl. 94. fig. 1. Stew. Elem. ii. 422. 'Purt. Brit. Faun. 201. Turt. Conch. Dict. 149.

Spirorbis communis, Fleming in Edin. Encyclop. vii. 68 ; and in Edin. Phil. Journ, xii. 245.

Spirorbis nautiloides, Lam. Anim. s. Vert. 2nde edit. v. 613. Stark, Elem. ii, 132. Templeton in Loud. Mag. N. Hist. ix. 233. Brown, İliust. pl. i. fig. 45. Griffith's Cuv, xiii. pl. 8. fig. 6. 
2. S. spirillum.

Serpula spirillum, Linn. Syst.1264. Fabric. Faun. Groenl. 376. Mont. Test. Brit. 499. Turt. Gmel, iv. 602. Turt. Brit. Faun. 200. Laskey in Wern. Mem. i. 412. Brown in ibid. ii. 534. Stew. Elem. ii. 421. Penn. Br. Zool. iv, 358. Turt. Conch. Dict. 150. Spirorbis spirillum, Flemiug in Edin. Encyclop. vii. 68; and in Edin. Phil. Journ. xii. 245. Lam. Anim. s. Vert. 2nde edit. v. 614. Stark, Elem. ii. 132. Templeton in Loud. Mag. N. Hist. ix. 233. Brown's Illust. pl. 1. fig. 41, 42, 53 and 54 .

3. S. granulatus.

Serpula granulata, Linn. Syst. 1266. Fabric. Faun. Groenl. 380. Mont. Test. Brit. 500. Laskey in Wern. Mem. i. 412. Brown in ibid. ii. 534. 'Turt. Brit. Faun. 201. Dillw. Rec. Sh. 1074. Penn. Brit. Zool. iv. 359. Turt. Conch. Dict. 150.

Serpula sulcata, Adams in Linn. Trans. iii. 255.

Spirorbis granulatus, Flem. in Edin. Encyclop. vii. 68; and in Edin. Phil. Journ. xii. 244. Templeton in Loud. Mag. N. Hist. ix. 233. Brown's Illust. pl. 1. fig. 47.

4. S. carinatus.

Serpula carinata, Mont. Test. Brit. 502. Dillw. Rec. Sh. 1074. Penn. Brit. Zool. iv. 360. Turt. Conch. Dict. 151. Turt. Br. Faun. 201.

Spirorbis carinatus, Fleming in Edin. Encyclop. vii. 68; and in Edin. Phil. Journ. xii. 244. Brown's Illust. pl. 1. fig. 48.

5. S. corrugatus.

Serpula corrugata, Mont. Test. Brit. 502. Penn. Brit. Zool. iv. 360. Turt. Brit. Faun. 201. Turt. Conch. Dict. 151. Dillw. Rec. Sh. 1075.

Spirorbis corrugatus, Fleming in Edin. Encyclop. vii. 68. pl. 204. fig. 13 ; and in Edin. Phil. Journ. xii. 245. Brown's Illust. pl. 1. fig. 46.

6. S. Montagui.

Serpula, new species, Mont. Test. Brit. 502.

Spirorbis Montagui, Fleming in Edin. Phil. Journ. xii. 245.

7. S. incurvatus.

Serpula incurvata, Turt. Gmel. iv. 609. Turt. Conch. Dict. 156. Dillw. Rec. Sh. 1071.

Vermiculum incurvatum, Mont. Test. Brit. 518. Turt. Br. Faun. 199.

Spirorbis incurvatus, Fleming in Edin. Phil. Journ. xii. 245.

8. S. pervius.

Vermiculum pervium, Mont. 'Test. Brit. 518.

Spirorbis pervius, Fleming in Edin. Phil. Journ. xii. 245.

Obs. By Turton and Dillwyn this is registered as a variety of the preceding.

9. S. retortus.

Serpula retorta, Turt. Gmel. iv. 609 Turt. Conch. Dict. 158. Penn. Brit. Zool, iv. 365. 
Vermiculum retortum, Mont. Test. Brit. 524.

Spirurbis retorta, Fleming in Edin. Phil. Journ. xii. 245.

10. S. annulus.

Spirorbis annulus, Brown's Illust. pl. 1. fig. 44.

11. S. heterocliticus.

Spirorbis heterocliticus, Brown's Illust. pl. 1. fig. 57.

12. S. striatulus.

Spirorbis striatulus, Brown's lllust. pl. 1. fig. 59.

**Heterodisca, Flem. Phil. Zool. ii. 602.

13. S. heterostrophus.

Serpula heterostropha, Mont. Test. Brit. 503. Turt. Brit. Faun. 201. Dillw. Rec. Sh. 1075. Penn. Brit. Zool. iv. 359. Turt. Conch. Dict. 151.

Spirorbis heterostrophus, Fleming in Edin. Encyclop. vii. 68. pl. 205. fig. 1. Brown's Illust. pl. 1. fig. 55.

Heterodisca heterostropha, Fleming in Edin. Phil. Journ. xii. 247.

14. S. minutus.

Serpula minuta, Mont. Test. Brit. 505. Turt. Brit. Faun. 200. Dillw. Rec. Sh. 1072. Penn. Br. Zool. iv. 359. Turt. Conch. Dict. 150.

Spirorbis minutus, Fleming in Edin. Encyclop. vii. 68.

Heterodisca minuta, Fleming in Edin. Phil. Journ. xii. 247.

15. S. conicus.

Spirorbis conicus, Fleming in Edin. Encyclop. vii. 68. pl. 205 . fig. 3. Brown's lllust. pl. 1. fig. 58.

Heterodisca conica, Fleming in Edin. Phil. Journ. xii. 247.

16. S. lucidus.

Serpula lucida, Mont. Test. Brit. 506. Laskey in Wern. Mem. i. 412. Brown in ibid. ii. 534. Turt. Br. Faun. 201. Penn. Br. Zool. iv. 360. Dillw. Rec, Sh. 1075. Turt. Conch. Dict. 151.

Serpula reflexa, Adams in Linn. Trans.

Spirorbis lucidus, Fleming in Edin. Encyclop. vii. 68. Brown's lliust. p. 1. fig. 56, 60 .

Serpula simistrorsa, Mont. Test. Brit. 504.

Heterodisca lucida, Fleming in Edin. Phil. Journ. xii. 247.

Obs. The Serpula sinistrorsa is made a variety of Serp. spirillum by Turton, Dillwyn and Pennant.

17. S. reversus.

Serpula reversa, Mont. Test. Brit. 508. Turt. Br. Faun. 201. Penn. Brit. Zool. iv. 361.

Spirorbis reversus, Fleming in Edin. Encyclop. vii. 69. Brown's lllust. pl. 1. fig. 52.

Heterodisca reversa, Fleming in Edin. Phil. Journ. xii. 248.

Ohs. Dillwyn and Turton make this a variety of their Serpula ver. micularis. 


\section{Serpula.}

Serpula, Fleming in Edin. Encyclop. vii. 67.-Serpula et Vermilia, Lam. Anim. s. Vert. v. 360 and 368.-Vermilia, Fleming, Phil. Zool. ii. 601; and in Edin. Phil. Journ. xii. 242.

1. S. intricata.

Serpula intricata, Linn. Syst. 1265. Turt. Gmel. iv. 603. Stew. Elem. ii. 422. Dillw. Rec. Sh. 1073.

Serpula vermicularis, Mull. Zool. Dan. iii. 9. tab. 86. fig. 9. (animal) Mont. Test. Brit. 509. Laskey in Wern. Mem. i. 413. Brown in ibid. ii. 534. Stew. Elem. ii. 422. Turt. Brit. Faun. 201. Fleming in Edin. Encyclop. vii. 67. Turt. Conch. Dict. 152.

Vermilia intricata, Fleming in Edin. Phil. Journ. xii. 242.

Serpula Mulleri, Berkeley in Loud. Mag. Nat. Hist. vii. 421.

2. S. vermicularis.

Tubus vermicularis, Ellis, Corall. pl. 38. fig. 2.

Serpula vermicularis, Linn. Syst. 1267. Penn. Brit. Zool. iv. 361. Stark, Elem. ii. 132. Berkeley in Loud. Mag. Nat. Hist. vii. 421. Templeton in ibid. ix. 233. Turt. Gmel. iv. 605. Brown's Illust. pl. 2. fig. 2, 3.

Serpula triquetra, Bast. Opusc. Subs. i. ii. 79. tab. 9. fig. 3. Mont. Test. Brit. 511. Stew. Elem. ii. 422. Fleming in Edin. Encyclop. vii. 67. Laskey in Wern. Mem. i. 413. Brown in ibid. ii. 534. Penn. Brit. Zool. iv. 361. Turt. Brit. Faun. 202.

Serpula contortuplicata, Cuv. Règ. Anim. iii. 190. Griffith's Cuv. xiii. 10. pl. 8. fig. 1 .

Vermilia vermicularis, Fleming in Edin. Phil. Journ. xii. 242.

Vermilia triquetra, Brown's Illust. pl. 2. fig. 1.

Amphitrite campanulata, Sowerby. Turt. Brit. Faun. 137. Penn. Br. Zool. iv. 91.-Amphitrite penicillus, Gmel. fide Cuv.

3. S. triquetra.

Serpula triquetra, Linn. Syst. 1265. Mont. Test. Brit. Suppl. 157. Sowerby, Gen. tab. fig. 2. (opt. !) Turt. Conch. Dict. 152. Berkeley in Loud. Mag. Nat. Hist. 421. Turt. Gmel. iv. 603.

Vermilia triquetra, Lam. Anim. s. Vert. 2nde edit. v. 633. Stark, Elem. ii. 131. Templeton in Loud. Mag. Nat. Hist. ix. 233. Brown's Illust. pl. 2. fig. 5 .

Var. a. Lid testaceous and conical. Vermilia conica, Fleming in Edin. Phil. Journ. xii. 243.-Patella extinctorium, Turt. Conch. Dict. 138.

Var. $\beta$. Lid testaceous, armed with two or three spines. Serpula vermicularis, Zool. Dan. tab. 86. fig. 8. Cuv. Règ. Anim. iii. 191. -Vermilia armata, Fleming, sup. cit. 243.-Patella tricornis, Turt. Conch. Dict. 139.

Var. $\gamma$. Lid with a produced central forked process. Mont. Test. Brit. Suppl. 157. No. 4. Vermilia triquetra, Fleming in Edin. Phil. Journ. xii. 242.-Serpula triquetra, Sowerby, Brit. Misc. i. 63. tab. 31 . 
Var. $\delta$. Lid smooth and slightly concave. Mont. Test. Brit. Suppl. 157. No. 1.

See also the observations of J. B. Harvey in Charlesw. Mag. i. 477.

4. Serpula contortuplicata, Linn. Syst. 1266. Templeton in Loud. Mag. Nat. Hist. ix. 233 . Turt. Gmel. iv. 604.

Obs. 'Turton makes this his var. B. of Serpula vermicularis. The character of it given by Linnæus is very applicable to some varieties of S. triquetra.

Serpula contortus, Brown's Illust. pl. 2. fig. 4. S. spiralis, ibid. fig. 6, and S. perversa, ibid. fig. 7, are common states of Serpula triquetra, found on Sertularice and other corallines.

5. S. serrulata.

Serpula serrulata, Fleming in Edin. Encyclop. vii. 67. pl. 204. fig. 8, copied in Brown's Illust. pl. 2. fig. 8.

Vermilia serrulata, Fleming in Edin. Phil. Journ. xii. 243.

Vermilia tricuspidata, Morris, Cat. Br. Foss, p. 67.

6. Serpula filiformis.

"Serpula filiformis, Rees, Cyclop. vol. 10." Templeton in Loud. Mag. Nat. Hist. ix. 233. Mr. Morris mentions a fossil Serpula filiformis, Cat. Brit. Foss. p. 66. The species intended by Templeton may be Filograna implexa?

7. Serpula rugosa.

Serpula rugosa, Turt. Conch. Dict. 154.

\section{Filograna.}

Filograna, Berkeley in Zool. Journ. v. 427.-Filipora, Flem. Brit. Anim. 530.

1. Filograna implexa.

Filograna, Planc. Conch. 46.

Serpula corallifica, Pall. Elench. Zooph. 239.

Serpula filograna, Linn. Syst. 1265. Berkeley in Zool. Journ. iii. 230.

Serpula complexa, Turt. Conch. Dict. 153.

Tubipora fascicularis, Stew. Elem. ii. 426.

Filipora filograna, Flem. Brit. Anim. 530.

Filograna implexa, Berkeley in Zool. Journ. v. 427.

Serpula minima? Lam. Anim. s. Vert. 2nde edit. v. 625 . Templeton in Loud. Mag. Nat. Hist. ix. 233.

\section{Ditrupa.}

Ditrupa, Berkeley in Zool. Journ. v. 426.

1. D. subulata.

Dentalium subulatum, Deshayes in Zool. Journ. iv. 194.

Ditrupia subulata, Berkeley in Zool. Journ. v. 425-6. Morris, Cat. Br. Fuss. p. $6 \bar{j}$.

Ob̆s, Mr. Berkeley, at one time, was led to refer the Dentalium 
gadus of Montagu to this genus (Zool. Journ. v. 425), but this location remains to be proved. Vide Corrigenda ad finem of the above vol. of the Zool. Journal.

\section{Family Arenicolide.}

Les Arenicoles, Cuv. Règ. Anim. iii. 197.-Telethusa, Latr. Fam. Nat. 244.-Arenicoliens, Aud. and M. Edw. Litt. de la France, ii. 282.-Arenicolide, Johnston in Loud. Mag. Nat. Hist. viii. 566.

\section{Arenicola.}

Arenicola, Lam. Anim. s. Vert. v. 335. Schweigg. Handb. 594. Aud. and $M$. Edw. sup. cit. 283. Leach in Suppl. Encyclop. Brit. 452 .

1. A. piscatorum.

For the synonyms see Loud. Mag. Nat. Hist. viii. 567; and add Arenicola piscatorum, Edin. Journ. Nat. and Geogr. Sc. iii. 245. Aud. and M. Edw. Litt. de la France, ii. 285. pl. 8. fig. 8-12.

Arenicola marina, Templeton in Loud. Mag. Nat. Hist. ix. 234.

Lumbricus marinus, Rathke in Zool. Dan. iv. 39. pl. 155. fig. B. $1-4$.

2. A. ecaudata.

Arenicola ecaudata, Johnston in Loud. Mag. Nat. Hist. viii. 566. fig. 54.

\section{Tribe Nereidina.}

(MacLeay in Ann. Nat. Hist. iv. 385.)

Nereides vagce, Pallas, Misc. Zool. 113.-Dorsibranches, Cuv. Règ. Anim. iii. 197.-Annelides antennées, Lam. Anim. s. Vert. v. 302.Notobranchia, Latr. Fam. Nat. 238.-A. errantes, Aud. and M. Edwards, Litt. de la France, ii. 27. (See also Johnston in Ann. Nat. Hist. ii. 424.)

\section{Family EUNICA.}

Les Eunices, Cuv. Règ. Anim. iii. 199.-Eunica, Lam. Anim. s. Vert. v. 320.-Eunicea, Latr. Fam. Nat. 239.-Euniciens, Aud. and M. Edw. Litt. de la France, ï. 131.

\section{Eunice.}

Leodice, Lam. Anim. s. Vert. v. 322.-Eunice, Schweigg. Handb. 597.-Aud. and M. Edw. Litt. de la France, ii. 137.

1. E. sanguinea.

Nereis sanguinea, Montagu in Linn. Trans. xi. 20. tab. 3. fig. 1.

Leodice sanguinea, Iam. Anim. s. Vert. 2nde edit. v. 563. Stark, Elem. ii. 137.

Eunice sanguinea, Aud. and M. Edw. Litt. de la France, ii. 147.

2. E. pinnata.

Nereis pinnata, Mull. Zool. Dan. i. 31. tab. 29. fig. 4-7. Aud. and M. Edw. Litt. ii. 145. Fleming in Edin. Phil. Journ. ix. 254. 


\section{Onuphis.}

Onuphis, Aud. and M. Edw. Litt. de la France, ii. 151.

1. O. tubicola.

Nereis tubicola, Mull. Zool. Dan. Prod. 2625. Zool. Dan. i. 18. tab. 18. fig. 1-6. Turt. Gmel. iv. 87. Aud. and M. Edw. Litt. de la France, ii. 154.

Onuphis tubicola, Johnston in Ann. and Mag. N. Hist. xvi. 6. Obs. "The first British specimens of the species recognised were dredged off Sana Island by Mr. G. C. Hyndman from forty fathoms. I exhibited the specimens at the Cork meeting of the British Association 1843." - W. Thompson.

\section{Family Neremes.}

Nereides, Lam. Anim. s. Vert. v. 310. Johnston in Ann. Nat. Hist. iii. 289.-Nereidea, Latr. Fam. Nat, 240.-Nereidiens, Aud. and M. Edw. Litt. de la France, ii. 179.

\section{Nereis.}

Nereis, Linn. Syst. 1085. Cuv. Règ, Anim. iii. 201. Aud. and M. Edw. Litt. de la France, ii. 184 . Johnston in Ann. Nat. Hist. iii. 289, and v. 168.-Lycoris, Lam. Anim. s. Vert. v. 311.

1. N. brevimanus.

Nereis brevimanus, Johnston in Ann. Nat. Hist. v. 170.

2. $N$. viridis.

Nereis viridis, Johnston in loc. cit. 171.

3. N. pelagica.

Nereis pelagica, Johnston in Ann. Nat. Hist. $\nabla .172$.

4. N.bilineata.

Nereis bilineata, Johnston in lib. cit. iii. 295.

5. N. Dumerillii.

Nereis Dumerillii, Johnston in lib. cit. $\nabla .174$.

6. N. fucata.

Nereis fucata, Johnston in lib. cit. v. $\$ 75$.

7. N. renalis.

Nereis renalis, Johnston in lib. cit. v. 176.

8. N. longissima.

Nereis longissima, Johnston in lib. cit. $\nabla .178$.

9. N. margaritacea.

Nereis margaritacea, Johnston in Ann. Nat. Hist. iii. 294.

10. N. pulsatoria.

Nereis pulsatoria, Montagu. Aud. and M. Edw. Litt. de la France, ii. 194. pl. 4.f. 8-13.

1. Nereis iricolor, Montagu, Linn. Trans, vii. 82. Penn. Brit. Zool. iv. 94. Turt. Br. Faun. 135. 
2. Nereis margarita, Montagu, Linn. Trans, vii. 82. Penn. Brit. Zool. iv. 94. Turt. Brit. Faun. 135.

3. Nereis lineata, Montagu, Linn. Trans. vii. 83. Penn. Br. Zool. iv. 95. Turt. Br. Faun. 135.

4. Nereis maculosa, Montagu in Linn. Trans, xi. 21. pl. 3. fig. 4.

5. Nereis rufa.

Nereis rufa, Penn. Brit. Zool. iv. 96. pl. 27, fig. 2, 3. Hogg's Stockton, 29.

6. Nereis mollis.

Nereis mollis, Linn. Syst. 1085. Turt. Gmel. iv. 86. Jameson in Wern. Mem. i. 557. Penn. Br. Zool. iv. 96. Bosc, Vers, i. 168.

7. Nereis octentaculata.

Nereis octentaculata, Montagu in Linn. Trans. vii. 84. Penn. Brit. Zool. iv. 95. Turt. Brit. Faun. 135.

8. Nereis punctata.

Nereis punctata, Encyclop. Méthod. Vers, tab. 56. fig. 19, 20 !

Desc. Body $\frac{6}{10}$ ths long, $\frac{1}{6}$ th broad, linear, nearly equal at both extremities. Back rounded, brown, marked with three rows of light-coloured circular spots arranged longitudinally, and with eight similarly coloured trans. verse lines placed at unequal distances. The middle series of these spots is the faintest; and besides the three dorsal there is another series on each side placed on the foot-like processes. Head small, quadrangular, corneous, pale, with four black eyes, two on each side and approximate. Anterior margin of the head furnished with five conical setaceous two-jointed tentacula, two on each side and one in the centre, which is the shortest and inferior. Mouth terminal, with a large projectile proboscis unarmed with any teeth. Feet forty on each side. Each foot is divided at the apex into two processes; the superior terminated with a very long setaceous filament, and furnished with a retractile brush of fine hairs; the inferior has three short setaceous filaments, and a large brush of equally fine hairs. The filaments are not retractile. Ventral surface brownish. Tail abrupt, terminated with two setæ like the lateral filaments. Anus terminal.

This is a beautiful worm, and often glows with a metallic lustre of a light blue reflected from its pale spots. Its motions are very rapid. I have seen only one specimen, and this was many years ago. I had no doubt of its being the Nereis punctata figured in the work referred to, but it is not the Nereis punctata of Muller. The species is not noticed by Audouin and Milne-Edwards ; nor am I able to refer it to any defined genus.

\section{Syllis.}

Syllis, Savigny in Cuv. Règ. Anim. iii. 203. Lam. Anim. s. Vert. v. 317. Aud. and M. Edw. Litt. de la France, ii. 204. (Vide Ann. Nat. Hist. xiii. p. 235.)

1. S. armillaris.

Nereis armillaris, Mull. Wurm. 150. tab. 9. fig. 1-5 ; copied in Encyclop. Méthod. pl. 55. fig. 13-17. Turt. Gmel. iv. 86. Bosc, Vers, i. 168. Johnston in Ann. and Mag. Nat. Hist. xv. 145. pl. 9. fig. 1, 2.

2. S. prolifera.

Nereis prolifera, Mull. Zool. Dan. ii. 15. tab. 52. fg. 5-9; copied 
in Encyclop. Méthod. pl. 56. fig. 12-15. Turt. Gmel. iv. 90. Bosc, Vers, i. 174. Aud. and M. Edw. in Ann. des Sc. Nat. xxix. 231. note 3 ; and Litt. de la France, ii. 209. Johnston in lib. cit. 146. pl. 9. fig. $3,4$.

3. S.? noctiluca.

Nereis noctiluca, Linn. Syst. 1085. Turt. Gmel. iv. 86. Penn. Brit. Zool. iv. 93. Turt. Br. Faun. 134. Stew. Elem. i. 390. Abildgaard in Zool. Dan. iv. 31. tab. 148. fig. A. 1-3. Aud. and M. Edw. Litt. de la France, ii. 209.

\section{Myriana.}

Myriana, Aud. and $M$. Edw. Litt. de la France, ii. 216.

1. M.? pennigera.

Nereis pennigera, Montagu, Linn. Trans. ix. 111.tab.6. fig.3. Penn. Brit. Zool. iv. 95. Aud. and M. Edw. Litt. de la France, ii. 219.

\section{Phyllodoce.}

Phyllodoce, Cuv. Règ. Anim. iii. 202. Lam. Anim. s. Vert. v. 316. $A u d$. and $M$. $E d w$. Litt. de la France, ii. 219. Johnston in Ann. Nat. Hist. iv. 224.

1. P. lamelligera.

Phyllodoce lamelligera, Johnston in Ann. Nat. Hist. iv. 225. pl. 6. fig. 1-6.

2. P. maculata.

Phyllodoce maculata, Johnston in Ann. Nat. Hist. iv. 227. pl. 7. fig. 1-3.

3. P. bilineata.

Phyllodoce bilineata, Johnston in Ann. Nat. Hist. iv. 227. pl. 6. fig. 7-10.

4. P. viridis.

Phyllodoce viridis, Johnston in lib. cit. iv. 228. pl. 6. fig. 11-15.

Psamathe.

Psamathe, Johnston in Loud. Mag. Nat. Hist. ix. 14; and in Ann. Nat. Hist. iv. 229.

1. P. fusca.

Psamathe fusca, Johnston in Loud. Mag. ix. 15. fig. 1 ; and in Ann. Nat. Hist. iv. 230. pl. 7. fig. 4.

\section{Ioida.}

Ioida, Johnston in Ann. Nat. Hist. iv. 231.

1. I. macrophthalma.

Ioida macrophthalma, Johnston in Ann. N. Hist. iv. 231 pl. 7. fig. 5.

Nephtys.

Nephtys, Cuv. Règ. Anim. 203. Lam. Anim.s. Vert. v. 313. Aud. 
and M. Edw. Litt. de la France, ii. 232. Joknston in Loud. Mag. N. Hist. viii. 341 .

1. N. margaritacea.

Nephtys margaritacea, Johnston in Loud. Mag. Nat. Hist. viii. 341. fig. 33 .

Eollicita.

Pollicita, Johnston in Ann. and Mag. N. Hist. xvi. 4.

1. P.peripatus.

Bebryce peripatus, Johnston, Mr. Thompson's Rep. 273.

Pollicita peripatus, Johnston in Ann. and Mag. N. Hist. xvi. 5. pl.2. fig. 1-6.

\section{Glycera.}

Glycera, Lam. Anim. s. Vert. v. 314. Aud. and M. Edw. Litt. de la France, ii. 241.

1. G. alba.

Nereis alba, Mull. Zool. Dan. Prod. 217. Zool. Dan. ii. 29.tab.62. fig. 6, 7. Turt. Gmel. iv. 89. Aud. and M. Edw. Litt. ii. 243. Bosc, Vers, i. 172. Johnston in Ann, and Mag. Nat. Hist. xv. 147, pl. 9. fig. 1-9.

\section{Family Ariciads.}

Ariciens, Aud. and M. Edw. Litt. de la France, ii. 252.-Ariciadce, Johnston in Mag. Zool. and Bot. ii. 63.

\section{Leucodore.}

Leucodore, Johnston in Mag. Zool. and Bot. ii. 66.

1. L. ciliatus.

Leucodore ciliatus, Johnston in Mag. Zool. and Bot. ii. 67. pl. 3. fig. 1-6.

Obs. In Griffith's 'Cuvier' (vol. xiii. Annelida, pl. 4. fig. 2) there is a figure of our Leucodore ciliatus under the name of Spio seticornis. It is not said from what work this figure is copied. I cannot reconcile the description of the Spio seticornis of authors in general with the worm before us.

\section{Spio seticornis.}

Nereis minima tentaculis longissimis, Bast. Opusc. Subs, ii. 134. tab. 12. fig, 2.

Spio seticornis, Furt. Gmel. iv. 81. Turt. Brit. Faun. 137. Penn. Brit. Zool. iv. 92. Stark, Elem, ii. 138.

2. Spio crenaticornis.

Spio crenaticornis, Montagu in Linn. Trans. xi. 199. tab. 14. fig. 6. Spio quadricornis, Lam. Anim. s.Vert. 2nde edit. v. 559. Stark, Elem. ii. 138.

3. Spio calcarea.

Spio calcarea, Templeton in Loud. Mag. Nat. Hist. ix. 234. fig. 27. Obs. Probably the same as Spio seticornis. 
Nerine.

Nerine, Johnston in Mag. Zool. and Bot. ii. 68.

1. N. vulgaris.

Nerine vulgaris, Johnston, Mag. Zool. and Bot. ii. 70. pl. 2. fig. 1-8. Spio vulgaris, Johnston in Zool. Journ. iii. 335 and 487.

2. $N$. coniocephala.

Spio viridis, Johnston in Zool. Journ. iii. 486.

Nerine coniocephala, Johnston in Mag. Zool. and Bot. ii. 70. pl. 2. fig. 9-13.

Obs. This species is nearly allied to the Lumbricus cirratulus of Delle Chiaje, Anim. s. Vert. Nap. iv. p. 196. tab. 64. fig. 16.

\section{Family Aphroditaces.}

Aphrodite, Pallas, Misc. Zool. 75.-Les Aphrodites, Cuv. Règ. Anim. iii. 206.-Aphroditce, Lam. Anim. s. Vert. v. 304.-Aphroditea, Latr. Fam. Nat. 239.-Aphrodisiens, Aud. and M. Edw. Litt. de la France, ii. 58.-Aphroditacee, Johnston in Ann. Nat. Hist. ii. 424.

Spinther.

Spinther, Joknston in Ann. and Mag. N. Hist. xvi. 8.

1. S. oniscoides, Johnston, lib. cit. 9. pl. 2. fig. 7-14.

Sigalion.

Sigalion, Aud. and M. Edw. Litt. ii. 103. Johnston in Ann. N. Hist. ii. 428 and 438.

1. S. Boa.

Sigalion Boa, Johnston in Ann. N. Hist. ii. 439.

\section{Pholoë.}

Pholoë, Johnston in Ann. N. Hist, ii. 428.

1. P. inornata.

Pholoë inornata, Johnston in Ann. N. Hist. ii. 437.

Polynö.

Polynoë, Lam. Anim. s. Vert. v. 308 . Aud. and M. Edw. Litt. de la France, ii. 74. Johnston in Ann. N. Hist. ii. 428 and 431.Lepidonotus, Leack in Suppl. Encyclop. Brit: i. 452.

Obs. Dr. Leach's name has the claim of priority.

1. P. squamata.

Polynoê squamata, Johnston in Ann. Nat. Hist. ii. 432; and v. 307. Templeton in Loud. Mag. N. Hist. ix. 234.

Lepidonotus clavatus? Leach in Suppl. Encyclop. Brit. i. 452.

2. P. cirrata.

Polynoë cirrata, Johnston in lib. cit. ii. 434; and v. 307. (Ann. N. Hist. xvi. 183.) 
Obs. Halithaa clava, Templeton in Loud. Mag. N. Hist. ix. 23t, may not be distinct from this species.

3. P. impar.

Polynoë impar, Johnston in lib. cit. ii. 436.

4. P. viridis.

Polynoë viridis, Johnston in lib. cit. ii. 437.

5. P. scolopendrina.

Polynoë scolopendrina, Johnston in lib. cit. v. 307.

\section{Aphrodita.}

Aphrodita, Linn. Syst. 1084. Aud. and M. Edw. Litt. de la France, ii. 63. Leach in Suppl. Encyclop. Brit. i. 452. Johnston in Ann. N. Hist. ii. 427,-Halithea, Lam. Anim. s. Vert. v. 306.

1. A. aculeata.

Aphrodita aculeata, Johnston in Ann. N. Hist. ii. 429; and v. 305. - Templeton in Loud. Mag. N. Hist. ix. 234.

2. A. hystrix.

Aphrodita hystrix, Johnston in Ann. N. Hist. v. 305 ; and iv. 370.

3. A. borealis.

Aphrodita borealis, Johnston in lib. cit. iv. 370. pl. 10. fig. 1-13.

C. eruciformis.

\section{Species of Doubtful Place.}

Campontia.

Campontia eruciformis, Johnston in Zool. Journ. iii. 325 ; and iv. 421 ; and in Loud. Mag. N. Hist. viii. 179. fig. 18. M. Edwards in Lam. Anim. s. Vert. 2nde edit. v. 575 ; and in Litt. de la France, ii. 290.

Obs. MacLeay first suggested that this might be the larva of a dipterous fly, - a suggestion which Mr. Green thinks he has converted into a certainty (Charlesw. Mag. i. 279). But Mr. Green has evidently never seen our Campontia, and his conclusion is hence of no weight.

\section{Branchiarius.}

Branchiarius, Montagu in Linn. Trans. xi. 202.

1. B. quadrangularis.

Branchiarius quadrangularis, Montagu in Linn. Trans. xi. 202. pl. 14. fig. 1.

\section{Diplotis.}

Diplotis, Montagu in Linn. Trans. xi. 203.

1. D. hyalina.

Diplotis hyalina, Montagu in Linn. Trans, xi. 203. pl. 14. fig. 5. 
Derris.

Derris, Adams in Linn. Trans. ii. 67.

1. D. sanguinea.

Derris sanguinea, Adams in Linn.Trans. ii. 67. tab. 13. fig. 1, 2. Turt. Gmel. iv. 108. Turt. Brit. Faun. 132. Penn. Br. Zool. iv. 101.

XLVI.-Journey through Java, descriptive of its Topography and Natural History. By Dr. Fr. JunghunN*.

[Continued from p. 332.]

\section{Journey to the Merapi.}

We ascended from the Sawungang towards Andong, and at a height of 3000 feet came to a district which was covered with Saccharum Klaga growing to a height of from 15 to 20 feet. The forests then again appeared which had already been passed lower down. Here begins a frightful wilderness: high vaulted trees, covering the whole country far and wide, rose up from the deepest clefts and pressed against the steepest acclivities: climbers and densely interwoven shrubs filled up all the interstices between the stems. One while we came to a narrow mountain ridge scarcely. two feet broad, between steep disrupted masses of rock covered with trees; then we mounted up these steep acclivities, climbing from the stem of one tree to another; then, again, we found ourselves in deep, moist, rocky clefts, vaulted over by the foliage of the trees and shrubs so thickly that not a ray of the sun could penetrate to us. The clouds had settled low on the mountains, and enveloped us in their damp and cold mists, which brought with them a peculiar odour. These deep forests are formed of hundreds of species of trees, which belong to the most various families. Preeminent are the species of Ficus, easily distinguished by their white, tenacious, milky sap, which flows from the injured bark; and next to these, the Magnoliacee and Urticece. In the thicket which fills up the spaces between their gigantic stems, the beautiful flowers of species of Medinella and other Melastomacee shine forth; and Scitaminee (Amomum, Zingiber, \&c.) raise their luxuriant leaves to a height of 20 feet, whilst their variegated cones of blossoms only half appear above the moist ground. Urtica? dichotoma, Bl. 'Bydragen,' a small tree with beautiful leaves which on their under surface have white and parallel veins, adorns these thickets†. A little higher up occurs a beautiful social Lycopodium, which attains a height of scarcely three feet, and covers the moist parts of the woods, like our mosses, as a kind of coherent cushion.

* From the Botanische Zeitung, Sept. 5th and 12 th, 1845.

+ Arbor est elegans, trunco gracili, $30-40$ pedes alto, cinereo, ramisque gracilibus; foliis in ambitu ramulorum collectis.- Silvulas constituit visu singulares, declivia montis Merapi ex altitudine 4000 pedum ad 6000 tegentes.-Trunci, quo magis in altum montis adscendunt, eo humiliores evadunt, denique vix 20 pedes alti, Usneis tecti, e ramis longe dependentibus. 